\title{
A Semantic Web Platform for Online Vaccine Sentiment Surveillance
}

\author{
Arash Shaban-Nejad ${ }^{\star 1,2}$, Sonia Menon² and David Buckeridge ${ }^{2}$ \\ 'University of California, Berkeley, CA, USA; ${ }^{2}$ Clinical and Health Informatics Research Group, McGill University, Montreal, QC, \\ Canada
}

\section{Objective}

This paper presents our approach on design and development of an integrated semantic platform to capture the domain knowledge on vaccine sentiments, beliefs, and behaviours using ontologies. The vaccine sentiment ontology (VASON) provides more structure around the vast amount of unstructured data scattered over blog posts to facilitate blog content analysis, and discovering patterns of words or phrases in blogs text (e.g. specifying topics, themes, sentiment, beliefs and so on). It also assists in revealing opinionated claims and assertions in blogs and specifying the authors, forms, functions, geographical locations, audiences of blogs, as well as bloggers' motives.

\section{Introduction}

Real-time monitoring and analysis of vaccine concerns over time and location could help immunisation programmes to tailor more effective and timely strategies to address specific public health concerns. In recent years attempts $[1,2]$ are being made to develop a more systematic monitoring of broader public vaccine concerns resulting in vaccine refusals and potential disease outbreaks. Automated sentiment analysis software applications are being developed to detect and track the emergence and spread, geographically and temporally, of online social media reports on vaccines by developing a new application for opinion mining and sentiment analysis. Although many of the current approaches for automated sentiment analysis provide a timely method to assess the sentiment of a population towards vaccination, they do not assess beliefs, perceptions and behaviours. Incorporating semantic approach by using ontologies captures the domain knowledge and supports automated extraction and analysis of text in blog posts related to vaccination.

\section{Methods}

The Vaccine Attitude Surveillance using Semantic Analysis (VASSA) Framework [3] is intended to support the automated extraction and analysis of text in blog posts related to vaccination. The framework consists of a Natural Language Processing (NLP) module, which is used for text analysis and concept extraction, and the VASON Ontology, which models existing knowledge about the relationships between vaccination beliefs and behaviours. VASON is operating at the overlapping borders between three kingdoms, namely Health, Online social networks, and Human behavioural model. The health part deals with concepts such as vaccine, vaccination, disease, health states, and adverse event. Concepts such as blogs, blog posts, bloggers, comments, forum, web sites and media are grouped under social networking category, while we study human perception, awareness, belief, behaviour and sentiment under the behavioural classification. The interaction between these three major components are analysed with the human factor at the centre at both individual and population levels. The data for creating the VASON conceptual model comes from the literature, databases and some of the existing vocabularies and biomedical ontologies.

\section{Results}

The VASON ontology can be used to facilitate concept extraction and analysis of the extracted concepts using an NLP module. The development of the ontology is currently in progress and we are now performing several experiments for our text extraction using the VASON sub-taxonomies adapted/imported from the Vaccine Ontology(VO) and the Disease Ontology.

\section{Conclusions}

VASON aims to provide knowledge on the factors driving vaccine refusal and to identify potential interventions for increasing coverage. The work on the ontology is in progress, and the effort on integrating the ontological knowledge with existing relevant public health resources and services is still underway. Also we are enriching the semantic model to perform more sophisticated queries on the relations between vaccine beliefs, vaccine adverse events, and the risk factors.

\section{Keywords}

Vaccine Sentiment Analysis; Online Social Media; Vaccine refusal; Semantic Web; Ontologies

\section{Acknowledgments}

The project is funded by the Public Health Agency/Canadian Institutes of Health Research Influenza Research Network (PCIRN).

\section{References}

1. Salathé M., and Khandelwal S.: Assessing Vaccination Sentiments with Online Social Media: Implications for Infectious Disease Dynamics and Control . PLoS Comput Biol. Oct 2011; 7(10): e1002199.

2. Larson, H.J., Smith D.M.,Paterson P, et al. Measuring Vaccine Confidence. Lancet Infectious Diseases 2013; 13: 606 - 613. Infect Dis 2013 . doi.org/10.1016/S1473-3099(13)70108-7

3. Brien S., Naderi N., Shaban-Nejad A., Mondor L., Kroemker D., Buckeridge DL.: Vaccine attitude surveillance using semantic analysis: constructing a semantically annotated corpus. WWW (Companion Volume), ACM Press, 2013: 683-686.

\section{*Arash Shaban-Nejad}

E-mail: arash.shaban-nejad@mcgill.ca 